\title{
Risk factors for stuttering: a secondary analysis of a large data base
}

\author{
Vladeta Ajdacic-Gross · Stefan Vetter • Mario Müller • Wolfram Kawohl • \\ Franz Frey · Gianpiero Lupi - Anja Blechschmidt - Claudia Born • \\ Beatrix Latal $\cdot$ Wulf Rössler
}

Received: 24 February 2009/Accepted: 23 September 2009/Published online: 15 October 2009

(C) Springer-Verlag 2009

\begin{abstract}
The spectrum of risk and concomitant factors in stuttering is generally thought to be wide and heterogeneous. However, only a few studies have examined these factors using information from large databases. We examined the data on 11,905 Swiss conscripts from 2003. All cases with high psychiatric screening scores indicating "caseness" for a psychiatric disorder were excluded, among them potential malingerers, so that 9,814 records remained. The analyses rely on self-reported information about stuttering in childhood, problems at birth, problems in school, mental disorders of parents and relatives, childhood adversity and socio-demographic information. Statistical modelling was done using logistic regression and path analysis models. Risk factors determined in the logistic regression include premature birth, probable attention deficit hyperactive disorder, alcohol abuse of the parents, obsessive-compulsive disorder in parents and relatives, having a disabled mother and having a parent from a foreign country. There is no overwhelmingly strong risk
\end{abstract}

\author{
V. Ajdacic-Gross ( $\square)$ · W. Kawohl · W. Rössler \\ Research Unit for Clinical and Social Psychiatry, \\ Psychiatric University Hospital Zürich, Militärstr. 8, \\ PO Box 1930, 8021 Zurich, Switzerland \\ e-mail: vajdacic@dgsp.uzh.ch \\ S. Vetter · M. Müller \\ Centre for Disaster and Military Psychiatry, \\ University of Zurich, Zurich, Switzerland \\ F. Frey · G. Lupi \\ Medical Services Swiss Armed Forces, Ittigen, Switzerland \\ A. Blechschmidt · C. Born \\ University of Applied Sciences Northwestern Switzerland, \\ Basel, Switzerland \\ B. Latal \\ University Children's Hospital, Zurich, Switzerland
}

factor; all odds ratios are about 2 or below. In conclusion, large databases are helpful in revealing less obvious and less frequent risk factors for heterogeneous disorders such as stuttering. Obviously, not only secondary analyses, but also systematical large scale studies would be required to complete the complex epidemiological puzzle in stuttering. An extensive examination of young adults who were initially assessed in childhood might provide the most promising design.

Keywords Stuttering $\cdot$ Epidemiology $\cdot$ Risk factors

\section{Introduction}

Stuttering is a relatively common disorder of verbal fluency that emerges in childhood in about $5 \%$ of children [16]. It is typically manifested at the beginning of long words and complex sentences. The disorder may have serious social and psychological sequels for the person so affected, especially if it persists beyond adolescence, which is the case in one out of five persons with stuttering $[2,32]$. The empirical findings have thus far suggested some basic differentiations $[2,4,7,16]$. These include (1) distinguishing developmental, or idiopathic stuttering, from acquired stuttering, (2) distinguishing persistent stuttering from recovering stuttering, which occurs in about fourfifths of all cases, (3) distinguishing stuttering in the presence of family history of stuttering from stuttering where there is no family history; and finally, (4) distinguishing stuttering with comorbidity with other disorders from stuttering without comorbid conditions.

Since various brain areas and tracts are involved in the planning and producing of fluent speech [7, 15, 23, 32], a wide spectrum of etiological patterns and associated risk factors, correlates and intervention approaches, may be 
assumed. A wide range of possible risk factors has been proposed in literature including male sex, low intelligence scores, prenatal, perinatal or other brain damage, but also neurological lesions (particularly in stuttering without hereditary evidence), concussion and head injury, genetic risk, delayed speech development, attention deficit hyperactive disorder (ADHD) and associated symptoms (e.g. enhanced gross motor activity, impulsivity, decreased attentional focusing, decreased inhibitory control, perceptual sensitivity), developmental coordination disorder, learning disorders, emotionally reactive and sensitive temperament, neurotic conflicts and impaired child-parent interactions, negative parental reactions to normal childhood disfluency $[2-4,10,16,26]$.

It may reasonably be assumed that this list is far from complete. Till now, research into stuttering has only rarely made use of large epidemiological databases [14, 29] and challenging statistical models [21], which might provide valuable information on rare or less obvious risk factors. This paper aims to fill this gap by presenting secondary analyses carried out on a database of about 10,000 Swiss conscripts.

\section{Methods}

Sample

The sample comprises conscripts who attended recruitment to the Swiss Armed Forces in 2003. The Swiss Armed Forces introduced an all-new recruitment procedure for conscripts and female volunteers in stages during that year. A group of 14,157 individuals (out of 24,292 conscripts and 123 volunteers) underwent the new recruitment procedure, including a psychiatric screening that used a comprehensive questionnaire as its first step. After the exclusion of incomplete or erroneous records there were 12,523 records remaining among them 11,905 male conscripts aged 18-20. In the next step, all cases with high psychiatric screening scores were excluded, among them most potential malingerers who generally represent a major problem in conscription screening. The criteria for exclusion relied on the Symptom Checklist-90-Revised (SCL-90-R) caseness definition [13], and entailed either the Global Severity Index (GSI) score $\geq 63$ (after T-standardization) or two or more subscale scores $\geq 63$ (after T-standardization). Thus, further 2,091 data records were excluded from the analysis, whereas 9,814 records remained.

\section{Questionnaires}

The conscription procedure comprises, among other elements, a psychiatric screening. All screening sessions are introduced and supervised by military test psychologists.
The screening includes the SCL-90-R [12] as an externally validated psychometric instrument. Further questionnaires assess information on psychopathological symptoms, on substance use and other behavioural characteristics of the conscripts, on mental and school problems in childhood and youth, on childhood adversity issues, on mental disorders of parents and relatives, and additional demographic information. Regarding former mental and school problems the questionnaires assess stuttering ("Did you stutter in childhood?"), probable dyslexia ("Do you have problems with reading and writing?"), attention deficit hyperactive disorder ("Were you restless or fidgety at school?") and several other issues. Additional questions assess problems at birth ("Were there any complications at your birth?"), and spending the first days of life in an incubator.

All information relies on self-reporting by the conscripts. The use of anonymised information in epidemiologic studies was cleared by the Zurich State Ethical Committee (KEK) to fulfil all legal and data privacy protection exigencies.

Statistical modelling

For the preliminary bivariate analyses, we classified the putative correlates of stuttering either as putative risk factors (or putative indicators of risk factors), concomitant factors, and variables not unequivocally assignable as either risk or concomitant factor. In the subsequent multivariate analysis, i.e. logistic regression, we included only variables assigned as putative risk factors. Finally, we differentiated background (parental, family) risk factors, and intermediate risk factors in a path analysis model. The path analysis was based on logistic regressions for each intermediate risk factor. Irrelevant risk factors were excluded from the final model.

We analysed the data with common statistical models such as correlational and logistic regression analysis. Data compilation and initial analyses were performed using SAS for Windows (Version 8). The path analysis was performed with Mplus (Version 4.21).

\section{Results}

In the restricted sample, 408 men $(4.2 \%$ out of 9,814$)$ reported having stuttered in childhood. Without the elimination of malingerers and other SCL-90-R-high-scorers, this group would have contained 601 men $(5.1 \%$ out of 11,905).

Table 1 shows the results from the bivariate analyses. The upper part of the table represents putative risk factors (or putative indicators of risk factors), and the lower part the concomitant factors. In between are variables which are not unequivocally assignable to either risk or concomitant 
Table 1 Bivariate analyses of risk and concomitant factors of persisting or recovered stuttering in Swiss male conscripts 2003

\begin{tabular}{|c|c|c|c|c|}
\hline Variable & $\begin{array}{l}\text { Overall } \\
N\end{array}$ & $\begin{array}{l}\text { Stuttering } \\
N\end{array}$ & OR & $\begin{array}{l}\text { Confidence } \\
\text { interval }\end{array}$ \\
\hline \multicolumn{5}{|l|}{ Putative risk factors } \\
\hline Troubles at birth & 1,091 & 65 & 1.55 & $1.17-2.03$ \\
\hline Incubator & 1,012 & 61 & 1.56 & $1.18-2.06$ \\
\hline Restless and fidgety in school & 1,831 & 105 & 1.54 & $1.22-1.93$ \\
\hline Mother alcoholic & 88 & 10 & 3.00 & $1.54-5.84$ \\
\hline Father alcoholic & 305 & 25 & 2.13 & $1.39-3.24$ \\
\hline Mother with other addiction & 202 & 8 & 0.95 & $0.46-1.93$ \\
\hline Father with other addiction & 299 & 18 & 1.50 & $0.92-2.43$ \\
\hline Anxiety in family members/relatives & 324 & 21 & 1.63 & $1.03-2.56$ \\
\hline Compulsion in family members/relatives & 254 & 19 & 1.91 & $1.18-3.07$ \\
\hline Depression in family members/relatives & 1,067 & 44 & 0.99 & $0.71-1.36$ \\
\hline Schizophrenia in family members/relatives & 605 & 23 & 0.91 & $0.58-1.39$ \\
\hline Family member was psychiatric inpatient & 554 & 28 & 1.24 & $0.83-1.84$ \\
\hline Mother is disabled ${ }^{\mathrm{a}}$ & 114 & 12 & 2.76 & $1.50-5.06$ \\
\hline Father is disabled $^{\mathrm{a}}$ & 194 & 14 & 1.82 & $1.04-3.16$ \\
\hline Mother had a serious physical disease & 262 & 19 & 1.84 & $1.14-2.96$ \\
\hline Father had a serious physical disease & 307 & 20 & 1.64 & $1.02-2.60$ \\
\hline Parent from a foreign country & 1,842 & 98 & 1.39 & $1.09-1.75$ \\
\hline \multicolumn{5}{|l|}{ Risk or concomitant childhood factors } \\
\hline Sibling is disabled ${ }^{\mathrm{a}}$ & 188 & 6 & 0.76 & $0.33-1.71$ \\
\hline Sibling had a serious physical disease & 119 & 7 & 1.45 & $0.67-3.12$ \\
\hline Frequently beaten by parents or educators & 225 & 10 & 1.07 & $0.56-2.03$ \\
\hline Not grown up with both parents & 1,911 & 91 & 1.19 & $0.94-1.51$ \\
\hline Raised (at least for a while) in an asylum & 267 & 22 & 2.13 & $1.36-3.33$ \\
\hline Raised (at least for a while) with grandparents & 806 & 47 & 1.48 & $1.08-2.02$ \\
\hline Hospitalised because of head injury & 1,162 & 65 & 1.43 & $1.09-1.88$ \\
\hline \multicolumn{5}{|l|}{ Concomitant childhood factors } \\
\hline Came late to school ( 8 years or older) & 114 & 18 & 4.47 & $2.67-7.47$ \\
\hline Examined by school psychologist & 755 & 50 & 1.72 & $1.26-2.33$ \\
\hline Troubles with reading and writing & 474 & 57 & 3.50 & $2.59-4.70$ \\
\hline Teased for deformity or did not come to terms with deformity & 750 & 52 & 1.82 & $1.34-2.46$ \\
\hline Had psychological counselling and/or therapy & 749 & 45 & 1.53 & $1.11-2.10$ \\
\hline
\end{tabular}

Baseline $N$ : 9,814, persons with stuttering: 408, relevant odds ratios are shown in bold letters

${ }^{a}$ Since childhood or after an accident

factors. The results suggest that only small proportions of persons with stuttering are influenced by each putative risk or concomitant factor.

At first glance, several groups of risk factors can be differentiated:

- Birth-related problems, as indicated in approximately $10 \%$ of the conscripts, and $15 \%$ persons with a stuttering problem. The "incubator" item assigns premature births as a risk factor for stuttering. The odds ratios for both risk factors have a similar magnitude $(\sim 1.5)$.

- Having been restless and fidgety at school points to a probable ADHD background. Such behaviour was reported by one in four conscripts who reported also a stuttering condition

- Among the substance variables, both parents' alcohol variables yielded relevant odds ratios between 2 and 3, but not for the variables related to "other substance" disorders.

- Anxiety disorders and obsessive-compulsive disorders (OCD) of family members and other relatives had a noteworthy predictive effect, but no other disorders such as depression or schizophrenia. Also the "inpatient" item, which is a proxy for serious psychiatric disorders in family members and relatives, proved to be not relevant. 
- Disabling disorders and serious physical diseases of the parents yielded noteworthy odds ratios. The odds ratio of the item "disabled mother" is nearly as high as the odds ratio regarding mother's alcohol addiction.

- Having a parent from a foreign country slightly enhanced the risk of stuttering.

- Being raised separately from the parents includes items which need further analyses and information, because the temporal sequence of the effects is not obvious.

The concomitant variables (came late to school, saw the school psychologist, had difficulty with reading and writing, was teased for deformity, underwent psychological counselling and/or therapy) correspond to delayed intellectual development and to the psychosocial consequences of stuttering. Difficulty with reading and writing is a putative indicator for dyslexia, but possibly also secondary analphabetism.

In multivariate logistic regression analysis we included the risk factors and, in addition, the "frequently beaten" variable since we assumed that violence against the child typically had begun in its first years of life (Table 2). The number of relevant predictors decreased in multivariate analysis, however, the spectrum remained as broad as before. The variables that remained relevant after adjustment for the other factors are: incubator (i.e. premature birth), having been restless and fidgety at school (probable ADHD), alcohol abuse of the parents, OCD in family members and relatives, and having a parent from a foreign country.

The path analysis (Fig. 1; Table 3) helped to differentiate the intermediate risk factors from the background parental variables. Only a few background variables act simultaneously on stuttering and on any related intermediate variable. Alcohol abuse of the mother acts over three pathways: a direct pathway, an indirect pathway involving the incubator (premature birth) variable, and another indirect pathway involving having been "restless and fidgety at school" (probable ADHD). Since the latter variables are not associated, one may assume at least two different biological mechanisms representing the effects of alcohol abuse. The effects are similar to having a "parent from a foreign country" whose effect may be direct or mediated by the subject having been "restless and fidgety at school" (probable ADHD) or mediated by the more complex path leading over "frequently beaten" and "restless and fidgety at school" (probable ADHD). However, the fact that "frequently beaten" and stuttering are not interrelated clearly indicates two different mechanisms.

Moreover, the path analysis adds some perspectives on the risk factors themselves. Alcohol and substance abuse of the mother seem to be the most multifarious risk factor, but also "schizophrenia in family members/relatives" and having a "parent from a foreign country" are prominent.

\section{Discussion}

This study explored the spectrum of risk and concomitant factors of stuttering using data from a large population database. The data were derived from the psychiatric and psychological screening of Swiss conscripts in 2003, and
Table 2 Logistic regression analysis: risk factors of persisting or recovered stuttering in Swiss male conscripts 2003

Relevant odds ratios are shown in bold letters

\begin{tabular}{lcccc}
\hline Variable & Estimates & SE & OR & Confidence interval \\
\hline Troubles at birth & 0.25 & 0.15 & 1.28 & $0.95-1.72$ \\
Incubator & 0.35 & 0.15 & $\mathbf{1 . 4 1}$ & $1.04-1.90$ \\
Restless and fidgety in school & 0.36 & 0.11 & $\mathbf{1 . 4 4}$ & $1.14-1.81$ \\
Mother alcoholic & 0.77 & 0.37 & $\mathbf{2 . 1 6}$ & $1.04-4.46$ \\
Father alcoholic & 0.63 & 0.22 & $\mathbf{1 . 8 8}$ & $1.20-2.95$ \\
Mother with other addiction & -0.65 & 0.42 & 0.52 & $0.22-1.20$ \\
Father with other addiction & 0.28 & 0.27 & 1.32 & $0.76-2.29$ \\
Anxiety in family members/relatives & 0.42 & 0.24 & 1.52 & $0.93-2.47$ \\
Compulsion in family members/relatives & 0.60 & 0.25 & $\mathbf{1 . 8 2}$ & $1.10-3.01$ \\
Depression in family members/relatives & -0.19 & 0.17 & 0.82 & $0.58-1.16$ \\
Schizophrenia in family members/relatives & -0.35 & 0.23 & 0.70 & $0.44-1.11$ \\
Family member was psychiatric inpatient & 0.11 & 0.21 & 1.11 & $0.72-1.70$ \\
Mother is disabled & 0.66 & 0.34 & 1.93 & $0.98-3.78$ \\
Father is disabled & 0.29 & 0.31 & 1.33 & $0.72-2.47$ \\
Mother had a serious physical disease & 0.42 & 0.26 & 1.53 & $0.91-2.55$ \\
Father had a serious physical disease & 0.24 & 0.25 & 1.28 & $0.76-2.11$ \\
Parent from a foreign country & 0.29 & 0.12 & $\mathbf{1 . 3 4}$ & $1.05-1.69$ \\
Frequently beaten by parents or educators & -0.33 & 0.35 & 0.72 & $0.36-1.43$ \\
\hline
\end{tabular}


Fig. 1 Path analysis of background and intermediate risk factors of persisting or recovered stuttering in Swiss male conscripts 2003 (see also Table 3)

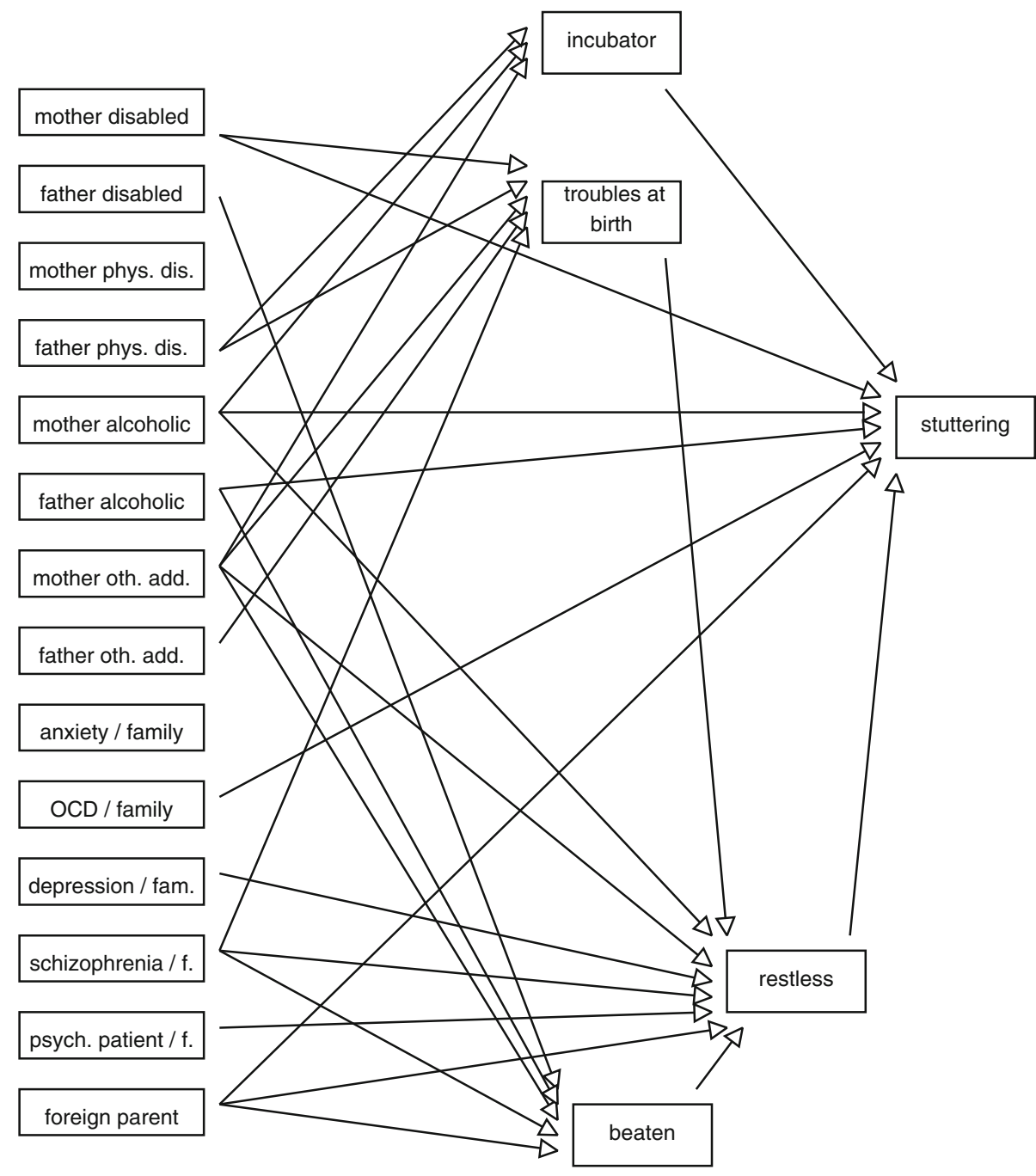

covered a broad spectrum of psychological items and psychiatric symptoms, among them rare risk and concomitant factors. We could identify a set of well-known correlates, as well as rare or new putative risk factors. Among the latter were premature birth, alcohol abuse of the parents, and having a parent from a foreign country.

\section{Methodological considerations}

As is common in secondary analyses of existing epidemiological data, this is a hypothesis-generating study. However, the context of mandatory military conscription delivers data, which may be quite different from the common context of a representative population-based study. Thus, we omitted all cases with high psychiatric symptom loads in order to avoid systematic bias that we might expect with regards to malingering. This equally resulted in omitting an unknown number of conscripts with valid responses, but high comorbidity. Provided that the major sources of systematic biasing effects (malingering, comorbidity) were excluded, the remaining source of bias, for example, self-reporting, are few. More importantly, they mainly yield noise in the data, and, as a consequence, smoothed instead of biased results. We have to expect that some of the effects that we found in this study are stronger in reality, and that we possibly missed some weak effects due to noisy data. Since we are dealing with risk factors, the consequences are not as grave as in studies focusing mainly on prevalence or incidence estimates.

\section{Interpretation of the results}

In accordance with the assumption that stuttering is a heterogeneous disorder, we found risk factors that obviously represented a wide spectrum of etiological mechanisms and related disturbances. There is no one overwhelming strong risk factor; all odds ratios are about 2 or below.

Having been "restless and fidgety in school" was an expected correlate of stuttering representing ADHD [2, 6]. 
Table 3 Path analysis of background and intermediate risk factors of persisting or recovered stuttering in Swiss male conscripts 2003

\begin{tabular}{|c|c|c|c|c|}
\hline Variable & Estimates & SE & OR & Confidence interval \\
\hline \multicolumn{5}{|l|}{ Incubator $\mathrm{ON}$} \\
\hline Mother alcoholic & 0.62 & 0.28 & 1.86 & $1.08-3.20$ \\
\hline Mother with other addiction & 0.49 & 0.19 & 1.63 & $1.12-2.39$ \\
\hline Father had a serious physical disease & 0.37 & 0.17 & 1.45 & $1.04-2.00$ \\
\hline \multicolumn{5}{|l|}{ Troubles at birth ON } \\
\hline Father had a serious physical disease & 0.54 & 0.15 & 1.72 & $1.28-2.32$ \\
\hline Father with other addiction & 0.48 & 0.16 & 1.62 & $1.17-2.23$ \\
\hline Mother with other addiction & 0.54 & 0.19 & 1.71 & $1.18-2.49$ \\
\hline Schizophrenia in family members/relatives & 0.32 & 0.12 & 1.38 & $1.09-1.75$ \\
\hline Mother is disabled & 0.60 & 0.24 & 1.83 & $1.14-2.93$ \\
\hline \multicolumn{5}{|l|}{ Frequently beaten by parents or educators $\mathrm{ON}$} \\
\hline Father is disabled & 0.74 & 0.34 & 2.09 & $1.07-4.08$ \\
\hline Father alcoholic & 1.60 & 0.21 & 4.95 & $3.29-7.44$ \\
\hline mother with other addiction & 1.30 & 0.27 & 3.68 & $2.18-6.20$ \\
\hline Schizophrenia in family members/relatives & 0.56 & 0.22 & 1.75 & $1.15-2.67$ \\
\hline Parent from a foreign country & 0.91 & 0.14 & 2.49 & $1.88-3.30$ \\
\hline \multicolumn{5}{|l|}{ Restless and fidgety in school ON } \\
\hline Troubles at birth & 0.35 & 0.08 & 1.42 & $1.22-1.65$ \\
\hline Mother alcoholic & 0.93 & 0.23 & 2.55 & $1.64-3.97$ \\
\hline Mother with other addiction & 0.51 & 0.16 & 1.66 & $1.22-2.27$ \\
\hline Depression in family members/relatives & 0.28 & 0.08 & 1.32 & $1.12-1.55$ \\
\hline Schizophrenia in family members/relatives & 0.35 & 0.10 & 1.42 & $1.17-1.72$ \\
\hline Family member was psychiatric inpatient & 0.29 & 0.11 & 1.34 & $1.08-1.65$ \\
\hline Parent from a foreign country & 0.30 & 0.06 & 1.35 & $1.19-1.53$ \\
\hline Frequently beaten by parents or educators & 1.02 & 0.14 & 2.77 & $2.10-3.65$ \\
\hline \multicolumn{5}{|l|}{ Stuttering ON } \\
\hline Incubator & 0.42 & 0.14 & 1.53 & $1.15-2.03$ \\
\hline Mother is disabled & 0.81 & 0.32 & 2.26 & $1.20-4.24$ \\
\hline Mother alcoholic & 0.73 & 0.36 & 2.07 & $1.02-4.20$ \\
\hline Father alcoholic & 0.61 & 0.22 & 1.84 & $1.19-2.85$ \\
\hline Compulsion in family members/relatives & 0.56 & 0.25 & 1.75 & $1.08-2.86$ \\
\hline Parent from a foreign country & 0.29 & 0.12 & 1.34 & $1.06-1.69$ \\
\hline Restless and fidgety in school & 0.36 & 0.12 & 1.43 & $1.14-1.81$ \\
\hline
\end{tabular}

ADHD has been suggested to be associated with other related disorders such as dyslexia [25]. The underlying common etiologies may be both of hereditary nature as well associated with acquired or secondary disorders [2].

OCD in family members and relatives and other parental psychological influences were a favourite topic of psychological stuttering research in the twentieth century, but do not remain so [4]. It is remarkable that no other psychiatric disorder of parents occurs in the list of risk factors in our large database. Thus, one might speculate that some pathways in OCD and in stuttering share a genetic and biological background. For example, basal ganglia circuits have been suggested as a possible link between stuttering and OCD [1], but also may be involved in ADHD [28].

Some relevant items found in our analysis have been infrequently reported. Premature birth has occasionally been presented as a risk factor for stuttering [2, 18, 20]. In contrast to other studies [8, 27], no effect on probable ADHD could be shown in the Swiss conscripts data. The missing link might be due to the fact that premature birth appears to be associated with attention problems rather than with hyperactivity problems [19]. Interestingly, the item "complications at birth" turned out to be relevant as regards probable ADHD but not as regards stuttering behaviour in multivariate analysis. Probably, the results were shaped by the fact that many participants equated premature births with problems at birth (about $40 \%$ redundant positive answers in each variable).

Alcohol abuse of the parents also needs further examination. Alcohol abuse has been rarely documented as a risk factor in stuttering [22]. Alcohol, but not other substances, seems to have a crucial effect on stuttering in our analysis. 
This indicates that the association is hardly related to adverse social environments, but rather to the foetal alcohol spectrum disorders (FASD). As we have no exact information about the parents' age of alcohol abuse, we may assume that misuse began during adolescence and young adulthood, which is typical of the initiation of substance misuse patterns. Alcohol misuse by the pregnant mother is known to have serious effects on the prenatal brain development leading to FASD [30, 31]. FASD are known to be associated with learning disabilities, lower IQ, ADHD, and motor impairment. Similar, eventually less severe consequences may also result from occasional alcohol misuse [9]. In contrast to the mother's alcohol abuse, alcoholism of the father as a risk factor for stuttering behaviour is more difficult to interpret. Possibly, this variable is a proxy for the alcohol misuse of the mother at lower exposure levels. An alternative explanation might suggest impulsiveness and aggressive behaviour by the alcoholic father as possible intermediate mechanisms. However, this is not supported by the variable "frequently beaten". As with other variables indicating adverse social environment, it proved not to be relevant with regards to stuttering.

Having a parent from a foreign country is an intriguing, although not a particularly strong, putative risk factor. The first hypothesis, impulsiveness and aggression deriving from the association with the variable "frequently beaten", was not supported by this analysis. Alternatively, "having a parent from a foreign country" might point at problems in language development in bilingual or multilingual families. A recent study has identified bilingual children to be at higher risk for stuttering than monolingual children or children speaking an alternative language exclusively [17].

"Having a disabled mother" is a risk factor which reappears in the list if a more narrow set of predictors was considered as is the case in the submodels within the path analysis. Since the path leading from "disabled mother" to "complications at birth" does not carry on to "stuttering", the focus should be on prenatal or postnatal factors. Unfortunately, no further information was available in our data which would have allowed us to be more specific.

Path analysis

The path analysis provides a more adequate perspective on the complex framework of background and intermediate variables involved in the prediction of a neurobehavioural symptom such as stuttering. Apart from risk factors generating direct effects on stuttering, there are also additional indirect paths. Comparing indirect paths may be helpful in revealing distinct mechanisms and processes leading to stuttering, even if they start with the same risk factor such as alcohol abuse of the mother.
Concomitant factors in stuttering

The associations with concomitant factors are in line with previous research. There is a close and well-known association between stuttering and dyslexia [5]. The odds ratios in this association, and with regard to delayed school start, are distinctly the strongest in our analysis. Having been teased for deformity and having had psychological counselling or treatment by the school psychologist or a psychotherapist are obvious psycho-social consequences of stuttering.

\section{Limitations}

This study relied on screening data, which were not specifically designed for the analysis of speech problems, such that various putatively interesting variables are missing in our analysis. In particular, it was not clear whether the results related to persistent stuttering or transient stuttering during childhood. More detailed information accessible in epidemiological surveys might include signs of stuttering [11], their persistence [5] and the differentiation from other speech disorders [24]. Moreover, no information was available in the Swiss conscripts data on stuttering in the family and among relatives. Some risk factors were represented by proxy variables such as premature birth ("incubator") and probable ADHD ("restless and fidgety in school"). The information relied on self-reporting of symptoms, problems and behaviour in a computer-assisted interview, and was therefore subject to recall bias. No clinical validation of any syndrome or any symptoms was available. The false-positive and false-negative self-reports have mostly a smoothing effect on the results.

\section{Conclusion}

Large databases are helpful in revealing some less obvious and less frequent risk factors for heterogeneous disorders, such as stuttering. Such factors found in this study include premature birth, alcohol abuse of the parents, or having a parent from a foreign country. Obviously, not only secondary analyses, but also systematical large scale studies, would be required to complete the complex epidemiological puzzle in stuttering. An extensive survey of young adults with stuttering and a control group, who were both initially assessed in childhood, might provide the most promising design. The survey should include detailed information on the neurological, psychiatric and other disabling disorders among the participants' parents and families. 


\section{References}

1. Alm PA (2004) Stuttering and the basal ganglia circuits: a critical review of possible relations. J Commun Disord 37:325-369

2. Alm PA, Risberg J (2007) Stuttering in adults: the acoustic startle response, temperamental traits, and biological factors. J Commun Disord 40:1-41

3. Anderson JD, Pellowski MW, Conture EG, Kelly EM (2003) Temperamental characteristics of young children who stutter. J Speech Lang Hear Res 46:1221-1233

4. Andrews G, Craig A, Feyer AM, Hoddinott S, Howie P, Neilson M (1983) Stuttering: a review of research findings and theories circa 1982. J Speech Hear Disord 48:226-246

5. Ardila A, Bateman JR, Nino CR, Pulido E, Rivera DB, Vanegas CJ (1994) An epidemiologic study of stuttering. J Commun Disord 27:37-48

6. Blood GW, Ridenour VJ, Qualls CD, Hammer CS (2003) Co-occurring disorders in children who stutter. J Commun Disord $36: 427-448$

7. Buchel C, Sommer M (2004) What causes stuttering? PLoS Biol 2:E46

8. Chapieski ML, Evankovich KD (1997) Behavioral effects of prematurity. Semin Perinatol 21:221-239

9. Coffin JM, Baroody S, Schneider K, O’Neill J (2005) Impaired cerebellar learning in children with prenatal alcohol exposure: a comparative study of eyeblink conditioning in children with ADHD and dyslexia. Cortex 41:389-398

10. Conture EG, Anderson JD, Pellowski MW (2001) Stottern in Theorie und Forschung: Träume der Theoriebildung treffen auf die empirische Wirklichkeit des Forschungsalltags. Sprache Stimme Gehör 25:1-9

11. Craig A, Hancock K, Tran Y, Craig M, Peters K (2002) Epidemiology of stuttering in the community across the entire life span. J Speech Lang Hear Res 45:1097-1105

12. Derogatis LR (1977) SCL-90. Administration, scoring and procedures Manual-1 for the R (revised) version and other instruments of the Psychopathology Rating Scale Series, Chigaco

13. Derogatis LR (1983) SCL-90. Administration, scoring and procedures Manual-2 for the R (revised) version and other instruments of the Psychopathology Rating Scale Series, Towson

14. Dworzynski K, Remington A, Rijsdijk F, Howell P, Plomin R (2007) Genetic etiology in cases of recovered and persistent stuttering in an unselected, longitudinal sample of young twins. Am J Speech Lang Pathol 16:169-178

15. Fox PT, Ingham RJ, Ingham JC, Zamarripa F, Xiong JH, Lancaster JL (2000) Brain correlates of stuttering and syllable production. A PET performance-correlation analysis. Brain 123:1985-2004
16. Howell P (2007) Signs of developmental stuttering up to age eight and at 12 plus. Clin Psychol Rev 27:287-306

17. Howell P, Davis S, Williams R (2009) The effects of bilingualism on stuttering during late childhood. Arch Dis Child 94:42-46

18. Jennische M, Sedin G (1999) Speech and language skills in children who required neonatal intensive care: evaluation at $6.5 \mathrm{y}$ of age based on interviews with parents. Acta Paediatr 88:975982

19. Johnson S (2007) Cognitive and behavioural outcomes following very preterm birth. Semin Fetal Neonatal Med 12:363-373

20. Largo RH, Molinari L, Kundu S, Lipp A, Duc G (1990) Intellectual outcome, speech and school performance in high risk preterm children with birth weight appropriate for gestational age. Eur J Pediatr 149:845-850

21. Levine SZ, Petrides KV, Davis S, Jackson CJ, Howell P (2005) The use of structural equation modeling in stuttering research: concepts and directions. Stammering Res 1:344-363

22. Löser H (1995) Alkoholembryopathie und Alkoholeffekte. G. Fischer, Stuttgart

23. Ludlow CL, Loucks T (2003) Stuttering: a dynamic motor control disorder. J Fluency Disord 28:273-295

24. McKinnon DH, McLeod S, Reilly S (2007) The prevalence of stuttering, voice, and speech-sound disorders in primary school students in Australia. Lang Speech Hear Serv Sch 38:5-15

25. Pennington BF (2006) From single to multiple deficit models of developmental disorders. Cognition 101:385-413

26. Poulos MG, Webster WG (1991) Family history as a basis for subgrouping people who stutter. J Speech Hear Res 34:5-10

27. Pringsheim T, Sandor P, Lang A, Shah P, O'Connor P (2009) Prenatal and perinatal morbidity in children with Tourette syndrome and attention-deficit hyperactivity disorder. J Dev Behav Pediatr 30:115-121

28. Schneider M, Retz W, Coogan A, Thome J, Rosler M (2006) Anatomical and functional brain imaging in adult attention-deficit/hyperactivity disorder (ADHD) - a neurological view. Eur Arch Psychiatry Clin Neurosci 256(1):i32-i41

29. Seery CH, Watkins RV, Mangelsdorf SC, Shigeto A (2007) Subtyping stuttering II: contributions from language and temperament. J Fluency Disord 32:197-217

30. Spohr HL, Willms J, Steinhausen HC (2007) Fetal alcohol spectrum disorders in young adulthood. J Pediatr 150:175-179

31. Steinhausen HC, Spohr HL (1998) Long-term outcome of children with fetal alcohol syndrome: psychopathology, behavior, and intelligence. Alcohol Clin Exp Res 22:334-338

32. Watkins KE, Smith SM, Davis S, Howell P (2008) Structural and functional abnormalities of the motor system in developmental stuttering. Brain 131:50-59 